\title{
Caracterización preliminar de los sitios de cría de Aedes (Stegomyia) albopictus (Skuse, 1894) (Diptera: Culicidae) en el municipio de Leticia, Amazonas, Colombia
}

\author{
José Joaquín Carvajal' ${ }^{1}$, Ligia Inés Moncada², Mauricio Humberto Rodríguez ${ }^{3}$, \\ Ligia del Pilar Pérez ${ }^{4}$, Víctor Alberto Olano ${ }^{5}$ \\ 1 Maestría en Estudios Amazónicos, Universidad Nacional de Colombia, Leticia, Colombia \\ 2 Departamento de Salud Pública, Facultad de Medicina, Universidad Nacional de Colombia, Bogotá, D.C., \\ Colombia \\ 3 The Manhiça Health Research Center, Maputo, Mozambique, África \\ 4 Secretaría de Salud Departamental del Amazonas, Leticia, Colombia \\ 5 Grupo de Entomología, Instituto Nacional de Salud, Bogotá, D.C., Colombia
}

Introducción. Dada la importancia de Aedes albopictus en la salud pública, es necesario caracterizar los criaderos para establecer medidas de control.

Objetivo. Caracterizar en función de los parámetros físico-químicos y grupos de organismos presentes, los criaderos de los estadios inmaduros de Ae. albopictus en Leticia, Amazonas.

Materiales y métodos. Se inspeccionaron 154 viviendas en el área urbana en diciembre 2002 y enero 2003, para buscar criaderos de Ae. albopictus y otros dípteros con estadios acuáticos inmaduros. En los criaderos con resultados positivos se tomaron datos físico-químicos cualitativos y cuantitativos: exposición al sol, ubicación, tamaño, material, conductividad, turbidez, oxígeno disuelto, temperatura y presencia de macroinvertebrados y plancton. Los resultados se compararon mediante análisis descriptivos, análisis de componentes principales, dendrogramas de clasificación e índices de diversidad.

Resultados. Se encontraron 21 criaderos con larvas de dípteros, 13 con Ae. albopictus; $92 \%$ de ellos estaban ubicados en el peridomicilio, en recipientes pequeños o medianos, dispuestos en la sombra, con baja turbidez y conductividad, bajos índices de diversidad para macroinvertebrados y altos para organismos productores de plancton. En el análisis de componentes principales, se encontró correlación significativa con ácaros, oligoquetos y hemípteros (macroinvertebrados), y con bacilarofíceas, clorofíceas y cianofíceas (plancton). En presencia de otros culícidos, las larvas de Ae. albopictus fueron escasas.

Conclusión. En este estudio se encontró que las hembras de Ae. albopictus depositan sus huevos en depósitos de agua recién establecidos con disponibilidad suficiente de recurso, baja conductividad y turbidez, y menor competencia intraespecífica e interespecífica.

Palabras clave: Aedes, vectores de enfermedades, control vectorial, plancton, Colombia.

Characterization of Aedes albopictus (Skuse, 1894) (Diptera:Culicidae) larval habitats near the Amazon River in Colombia

Introduction. Because the role of Aedes albopictus as an incriminated vector of several viral pathogens, its control is important to human health. To establish appropriate control methods, characterization of the larval habitats is a necessary first step.

Objective. Habitats of the immature stages of Ae. albopictus were characterized with respect to physical-chemical parameters and by floral and faunal arrays present.

Materials and methods. Leticia is located at the southernmost tip of Colombia on the banks of the Amazon River. In the urban area, 154 houses were inspected in December 2002 and January 2003. Physical-chemical data were collected, including exposure to sunlight, location, container size and material, water conductivity, and dissolved oxygen. Macroinvertebrates and plankton samples were taken at each positive larval site. The results were compared using descriptive analysis, principal component analysis, classification dendrograms, and diversity indexes. 
Results. Twenty-one habitats were found positive for Diptera, and 13 were positive for $A e$. albopictus larvae. Most of the positive habitats (92\%) were located near the houses--they were small or medium size receptacles located in the shade. This water generally had low conductivity and low turbidity, although high values of these parameters were also identified. The habitats had low diversity indexes for macroinvertebrates and high diversity indexes for plankton. In the principal component analysis, significant correlation was found with mites, oligochaetes and hemipterans (the macroinvertebrates) and with bacilarophyceaes, clorophyceaes and cianophyceas (the algal forms).

Conclusion. In Leticia, females of $A e$. albopictus were found in newly established habitats with sufficient availability of resources, low conductivity, and turbidity, lower intra-and interspecific competition.

Key words: Aedes, disease vectors, vector control, plankton, Colombia.

El mosquito Aedes (Stegomyia) albopictus fue identificado por primera vez en Suramérica en el estado de São Pãulo, Brasil en 1986 (1), y en Colombia en la Leticia, Amazonas, en 1998 (2). Posteriormente, se registró en Buenaventura (Valle del Cauca) en el 2001 (3) y en el 2007 en Cali (Valle del Cauca) (4).

Este mosquito es importante en salud pública por ser vector de los diferentes serotipos del virus del dengue en el sudeste asiático (5). En las Américas, además de este virus, potencialmente podría transmitir los virus de la fiebre amarilla y de la encefalitis equina venezolana, entre otros $(5,6)$. No obstante, hasta el presente no se le ha incriminado en la transmisión de agentes patógenos de importancia en salud pública en las Américas, aunque se han encontrado larvas de este mosquito infectadas con el virus del dengue (5-7) y adultos naturalmente infectados con el virus La Crosse (1) y el virus del oeste del Nilo (8). Méndez et al. (9) hicieron el primer reporte de infección natural de Ae. albopictus con los serotipos Den-1 y Den-2, en Colombia, de especímenes procedentes del municipio de Buenaventura, departamento del Valle del Cauca.

La presencia de Ae. albopictus vuelve más compleja la problemática del dengue, debido a

Correspondencia:

José Joaquín Carvajal, Maestría en Estudios Amazónicos, Universidad Nacional de Colombia, sede Leticia, Leticia, Colombia

Teléfono: (578) 592 7996, extensión: 109; fax (578) 592 7996, extensión 201.

jjcarvajalc@unal.edu.co

Recibido: 04/11/08; aceptado:31/03/09 la dificultad de control por su rápida colonización en áreas peridomésticas, lo cual constituye una amenaza porque podría ser la unión entre los ciclos de transmisión de la fiebre amarilla selvática y urbana (5).

Como componente de la vigilancia en salud pública, el sistema de vigilancia entomológica permite determinar los cambios en la distribución geográfica de los vectores, medir las variaciones de las poblaciones de formas inmaduras y adultas, identificar áreas con alta infestación, caracterizar sus sitios de cría y detectar la introducción de especies no nativas - la urbanización de especies silvestres, conocimiento básico para la toma de decisiones con el propósito de tomar medidas de control integrado y selectivo de vectores (10).

La caracterización de los criaderos acuáticos, hábitats de Ae. albopictus en sus fases inmaduras, permite identificar las características bióticas y abióticas, y las interrelaciones entre ellas en un hábitat particular, brindando una visión más amplia de la dinámica e importancia de los factores que intervienen en estos ecosistemas. Este conocimiento permite el entendimiento de su comportamiento biológico desde un enfoque ecosistémico, para proponer soluciones de manejo más eficientes para su control en el área de estudio.

En el presente trabajo se caracterizaron los criaderos de $A e$. albopictus en función de los parámetros físico-químicos y grupos de organismos presentes, en la zona urbana del municipio de Leticia (Amazonas), durante los meses de diciembre de 2002 y enero de 2003. 


\section{Materiales y métodos}

El área de estudio fue el casco urbano de Leticia, ciudad localizada a $04^{\circ} 12^{\prime} 55^{\prime \prime}$ sur y $69^{\circ} 56^{\prime} 26^{\prime \prime}$ oeste, con una altitud de 82 m.s.n.m. y una temperatura promedio de $27^{\circ} \mathrm{C}$. Tiene una extensión de $3.695 \mathrm{~km}^{2}$ y alrededor de 3.654 viviendas, con una población aproximada de 27.916 habitantes. Limita por el norte con Tarapacá, por el sur con la república de Perú, por el oeste con el municipio de Puerto Nariño y por el oriente constituye una sola área geográfica con la ciudad de Tabatinga, Brasil. El régimen de precipitación es monomodal; la temporada seca se presenta generalmente entre los meses de julio y septiembre y la lluviosa entre octubre y junio (11).

El muestreo se realizó en el período comprendido entre diciembre de 2002 y enero de 2003. Se muestrearon aleatoriamente 154 viviendas, con un error del $5 \%$ y una frecuencia esperada del índice de infestación de viviendas promedio (IIV) de 9,57\% (12). Las casas muestreadas se seleccionaron aleatoriamente mediante el programa Excel 2002 de acuerdo con una numeración preestablecida de las manzanas de la localidad. Se inspeccionaron el intradomicilio, el peridomicilio y el extradomicilio, en busca de criaderos potenciales con larvas, pupas o exhuvias de Ae. albopictus o de otros dípteros. En este trabajo, se tomó peridomicilio como el área comprendida desde el límite de la construcción hasta los $10 \mathrm{~m}$ y, el extradomicilio, a partir de los $10 \mathrm{~m}$.

\section{Toma de muestras}

Una vez localizado el criadero, se registró el tamaño según el volumen aproximado tomando área superficial y profundidad (menos de $1 \mathrm{~L}$ : pequeño; de 1 a $10 \mathrm{~L}$ : mediano, y más de $10 \mathrm{~L}$ : grande), bajo los siguientes criterios: textura, material, color, ubicación en el domicilio (intradomiciliario, peridomiciliario o extradomiciliario) y el tipo de exposición al sol (directa, parcial o sombra).

Se tomó una muestra de agua de $50 \mathrm{ml}$, aproximadamente, teniendo la precaución de no agitarla, con el fin de tomar el registro de turbidez, mediante un turbidímetro digital Hanna. Además, se tomaron registros del criadero de $\mathrm{pH}$, conductividad $(\mathrm{mS} / \mathrm{cm})$, oxígeno disuelto (mg/L), temperatura del agua $\left({ }^{\circ} \mathrm{C}\right)$, temperatura ambiental $\left({ }^{\circ} \mathrm{C}\right.$, mediante un termómetro manual) utilizando un conductímetro YSI 30, un oxímetro YSI 55 y un pHmetro WTW 330i/SET, los cuales registraban también la temperatura del criadero.

Para tomar las muestras de agua del criadero, se utilizó un cucharón de peltre esmaltado blanco de $250 \mathrm{ml}$ de capacidad y una pipeta plástica graduada con pera de caucho para los criaderos que, por su tamaño, no permitieran su medición, y así, estimar la cantidad aproximada de macroinvertebrados. La muestra se filtró en una red de poros de $0,01 \mathrm{~cm}^{2}$, para recolectar las larvas del mosquito y otros macroinvertebrados asociados. Las muestras se conservaron en alcohol al $70 \%$, en frascos de $100 \mathrm{ml}$. Los macroinvertebrados recolectados de cada muestra se trasladaron a cajas de Petri con alcohol al $70 \%$ para la determinación y cuantificación en esteroscopio Wild Heerbrugg o en microscopio Olympus CX31.

La muestra de plancton se tomó directamente y se envasó en un frasco de $100 \mathrm{ml}$, en una proporción 1:1 con transeau (agua destiladaalcohol etílico-formol, en proporción 6:3:1). En el laboratorio, las muestras de plancton se agitaron 50 veces, aproximadamente; se tomaron $5 \mathrm{ml}$ de cada muestra y se colocaron en piscinas de sedimentación por 10 horas (2 horas $/ \mathrm{ml}$ ). Posteriormente, se identificaron y cuantificaron evaluando la abundancia por cada campo en un microscopio óptico invertido Olympus CK22 ULWCD 0.30. Para la identificación del material biológico, se utilizaron las claves de Berrios y Sielfeld (13), McAlpine et al. (14), Merritt y Cummins (15), Milligan (16), Peckarsky et al. (17), Ospina (Ospina RT. Aspectos taxonómicos Chironomidae (Diptera). En: Memorias, Seminario Invertebrados Acuáticos y su Utilización en Estudios Ambientales. Bogotá: Universidad Nacional de Colombia y Sociedad Colombiana de Entomología; 1995), Thorp y Covich (18), y La Casse y Yamaguti (19). 


\section{Análisis de datos}

Los individuos de cada criadero se clasificaron por morfotipos hasta familia, género o especie, cuando fue posible. Se calcularon las abundancias relativas a partir de los datos extrapolados a una medida de volumen estándar. Los criaderos se clasificaron para el análisis en cuatro tipos, según presencia o ausencia de Ae. albopictus y otros dípteros. Se compararon los tipos de criaderos en relación con la composición, las abundancias relativas, la diversidad mediante los índices de Shannon-Wiener (diversidad), Simpson (dominancia), de uniformidad (equitativity) y de riqueza de especies.

Se aplicaron dos modelos estadísticos para análisis multivariado: un análisis de conglomerados basado en distancias euclideanas y el algoritmo UPGMA (unweighed pair group method with arithmetic mean), y un análisis de componentes principales con base en una matriz de datos de abundancia de morfotipos para cada clase de criadero, mediante el coeficiente de correlación $\rho$ de Pearson. Para el análisis del componente biológico, los datos se transformaron a logaritmo natural. Se utilizaron los paquetes estadísticos PAST, v1.73, Biodiversity Pro y Estimates Win800.

\section{Resultados}

En las 154 viviendas inspeccionadas se encontraron 21 criaderos con larvas de de dípteros, 13 (62\%) de ellos con Ae. albopictus. Estas larvas se distribuyeron por criadero, en la siguiente forma: A, exclusivamente con larvas de $A e$. albopictus; AC, larvas de Ae. albopictus y larvas de Chironominae sp. y Culex quinquefasciatus - Limatus durhamii; C, larvas de Chironominae sp. y $C x$. quinquefasciatus o Li. durhamii; y NA, exhuvias de pupas o larvas de culícidos diferentes a Ae. albopictus. La distribución general de los criaderos según las larvas encontradas en ellos fue: 3A (14\%), 10AC (48\%), 5C (24\%) y $3 \mathrm{NA}(14 \%)$.

El 95\% de las especies se encontraron en 17 de los 21 criaderos, lo que indica que las especies encontradas se distribuyeron ampliamente en todo el municipio. El $92 \%$ de los criaderos positivos para Ae. albopictus estaban ubicados en el peridomicilio, $77 \%$ eran latas y botellas, y $15 \%$, tanques bajos y una cáscara de coco.

\section{Parámetros físico-químicos cualitativos}

En las cuatro clases de criaderos, se encontraron recipientes plásticos (23\%) y metálicos (53\%), principalmente inservibles, y en los criaderos A, se encontró una cáscara de coco, tanques bajos y albercas. El blanco fue el color el más frecuente. En relación con la exposición al sol, se observó que los criaderos A y AC estaban en su mayoría a la sombra o con un área muy pequeña expuesta al sol; sin embargo, se encontraron dos criaderos $A C$ que estaban expuestos directamente a los rayos del sol. Los C tenían un área grande expuesta al sol y los NA estaban expuestos totalmente a los rayos del sol.

\section{Parámetros físico-químicos cuantitativos}

Ae. albopictus se encontró más frecuentemente en criaderos de tamaño pequeño y mediano, con oxígeno disuelto relativamente abundante, turbidez y conductividad bajas, pero toleraba baja concentración de oxígeno disuelto, y conductividad y turbidez altas (cuadro 1). En el análisis decomponentes principales, loscriaderos C se diferencian de los demás, principalmente, por su conductividad y volumen. La turbidez fue mayor en los criaderos AC y C (figura 1a). Además, como se muestra en el dendrograma (figura1b), los criaderos A y AC están agrupados y cercanos, y se separan claramente de los criaderos $C$ y NA. Los criaderos NA y A son de tamaño pequeño y se diferencian claramente en sus valores de conductividad; los A presentaron valores bajos y los NA, valores altos. En este estudio no se encontraron diferencias evidentes entre las clases de criaderos en la temperatura y el $\mathrm{pH}$.

\section{Macroinvertebrados}

La especie más abundante fue Ae. albopictus, que se encontró en $62 \%$ de los criaderos, seguida por Chironominae sp. con 45,5\%. En $23,8 \%$ de los criaderos estas dos especies se encontraron juntas, en $19 \%$ estaban Ae. albopictus, Chironominae sp., Cx. quinquefasciatus o $\mathrm{Li}$. durhamii, en 14,2\% había sólo larvas de $A e$. 
Cuadro 1. Valores máximos y mínimos de las condiciones abióticas registradas en cada tipo de criadero.

\begin{tabular}{|c|c|c|c|c|c|c|c|c|}
\hline \multirow[t]{2}{*}{ Variable } & \multicolumn{2}{|c|}{ A } & \multicolumn{2}{|c|}{$A C$} & \multicolumn{2}{|c|}{ C } & \multicolumn{2}{|c|}{ NA } \\
\hline & Min & Max & Min & Max & Min & Max & Min & Max \\
\hline Volumen (L) & 0,338 & 1,445 & 0,484 & 253,65 & 1,125 & 1680 & 2,7 & 24,64 \\
\hline $\begin{array}{l}\mathrm{T}\left({ }^{\circ} \mathrm{C}\right) \text { criadero } \\
\mathrm{T} \text { ambiental }\left({ }^{\circ} \mathrm{C}\right)\end{array}$ & 28,3 & 30,4 & 25 & 34,3 & 26,1 & 31,3 & 25,9 & 32,9 \\
\hline registrada in situ & 25 & 28,7 & 24 & 28,9 & 26,3 & 29,2 & 25,5 & 26,6 \\
\hline Saturación de O2 (\%) & 29 & 65 & 5,6 & 66,4 & 0,17 & 46,5 & 6,5 & 79,1 \\
\hline Oxígeno disuelto (mg/L) & 2,19 & 5,1 & 0,44 & 4,61 & 0,26 & 3,43 & 0,53 & 5,79 \\
\hline Turbidez (FTU) & 11 & 308 & 4 & 461 & 0 & 461 & 6 & 175 \\
\hline Conductividad mS/cm) & 51,6 & 118,8 & 15,17 & 291,3 & 70,9 & 459 & 79,8 & 239,5 \\
\hline $\mathrm{pH}$ & 7,15 & 8,46 & 6,39 & 8,82 & 5,66 & 7,24 & 6,53 & 7,88 \\
\hline
\end{tabular}

A, exclusivamente con larvas de Ae. albopictus; AC, larvas de Ae. albopictus y larvas de Chironominae sp. y Culex quinquefasciatus o Limatus durhamii; C, larvas de Chironominae sp. y Cx. quinquefasciatus o Li. durhamii; y NA, exhuvias de pupas o larvas de culícidos diferentes a Ae. albopictus.

a

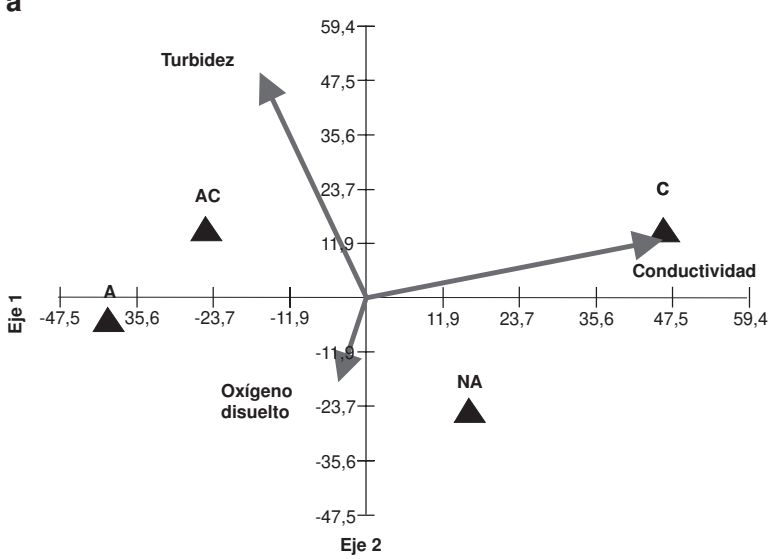

b

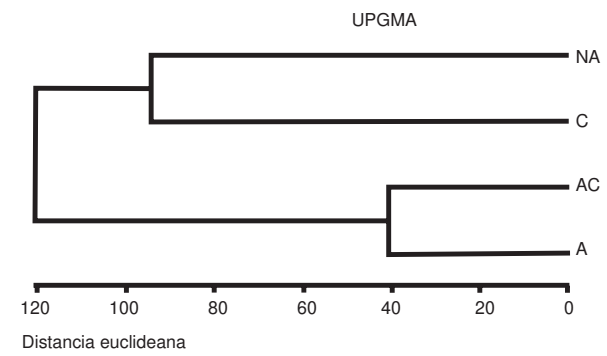

Figura 1. (a) Análisis de componentes principales a partir de parámetros físico-químicos cuantitativos. (b) Dendrograma de clasificación a partir de parámetros físico-químicos cuantitativos.

A, exclusivamente con larvas de Ae. albopictus; AC, larvas de Ae. albopictus y larvas de Chironominae sp. y Culex quinquefasciatus o Limatus durhamii; C, larvas de Chironominae sp. y Cx. quinquefasciatus o Li. durhamii; y NA, exhuvias de pupas o larvas de culícidos diferentes a Ae. albopictus.

albopictus y en 4,8\% Ae. albopictus se presentó junto con Li. durhamii. Es de anotar que $\mathrm{Li}$. durhamii presentó la mayor abundancia cuando se asoció con larvas de Ae. albopictus.

En el cuadro 2 se presentan las abundancias relativas de macroinvertebrados por clases de criaderos. En los criaderos AC, se encontró la mayor riqueza de especies y la mayor abundancia la mostraron $C x$. quinquefasciatus, Naididae, Ae. albopictus y Chironominae sp., respectivamente. Los criaderos NA no presentaron macroinvertebrados asociados.

Con el fin de esclarecer las estrategias de establecimiento del mosquito, se usaron índices ecológicos de diversidad que permiten visualizar de una manera más clara el papel de los morfotipos presentes en cada clase de criadero. En los criaderos A, los valores de diversidad y riqueza son bajos, lo que puede reflejar que son depósitos recién establecidos, mientras que los AC presentaban los valores mayores de riqueza, diversidad de Shannon-Wiener y uniformidad (equitivity), y el valor más bajo de dominancia (cuadro 3).

De acuerdo con el análisis de componentes principales, las poblaciones de Ae. albopictus se caracterizaron por estar correlacionadas significativamente con poblaciones de ácaros $(\rho=0,881, a=0,05)$, oligoquetos $(\rho=0,836$, a $=0,05)$ y hemípteros $(\rho=0,874, a=0,05)$, y no se 
Cuadro 2. Abundancias relativas de macroinvertebrados presentes por tipos de criaderos.

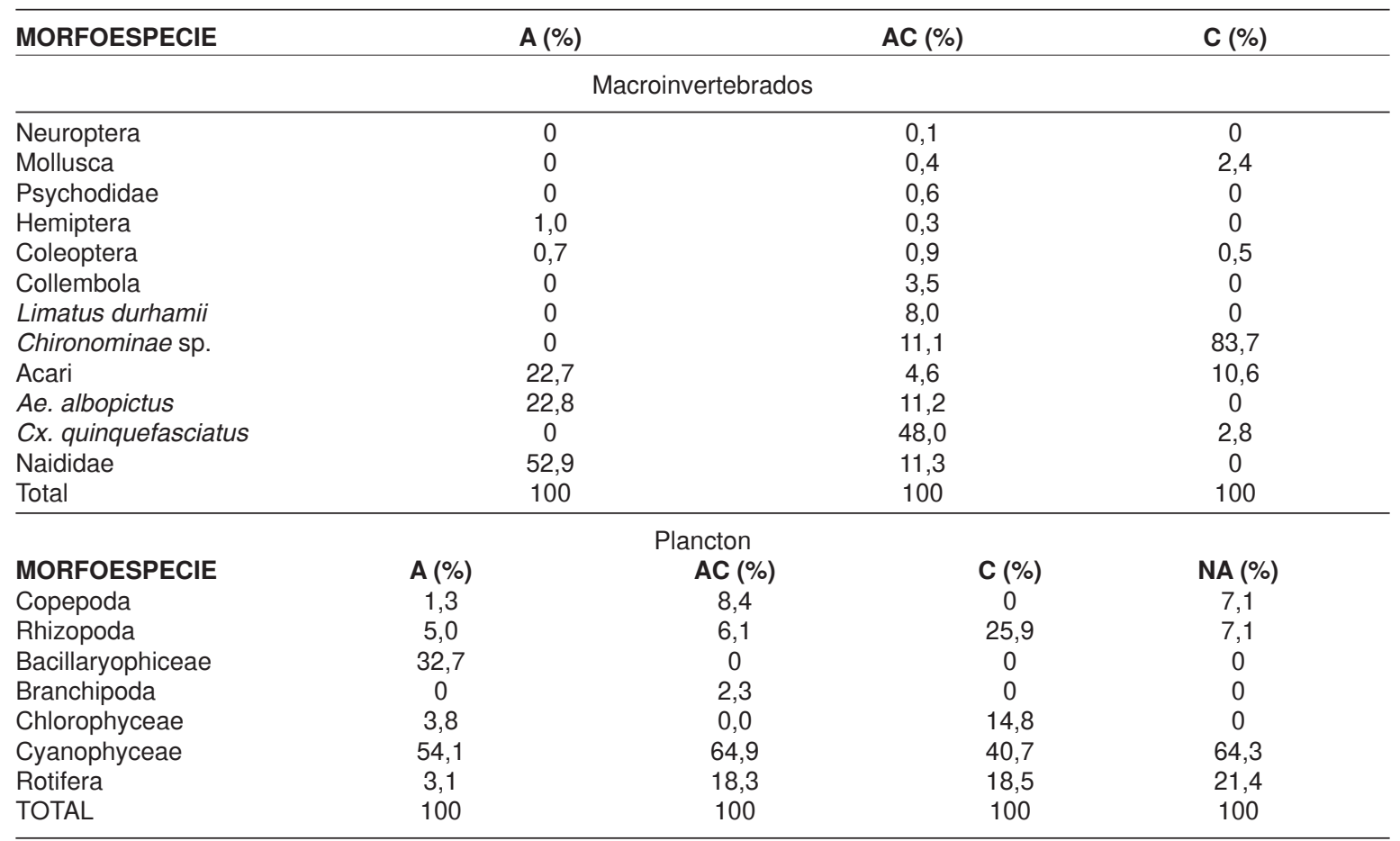

A, exclusivamente con larvas de Ae. albopictus; AC, larvas de Ae. albopictus y larvas de Chironominae sp. y Culex quinquefasciatus o Limatus durhamii; C, larvas de Chironominae sp. y Cx. quinquefasciatus o Li. durhamii; y NA, exhuvias de pupas o larvas de culícidos diferentes a Ae. albopictus.

Cuadro 3. Índices de diversidad por clases de criaderos a partir de las abundancias relativas de macroinvertebrados.

\begin{tabular}{lccc}
\hline Tipo de criadero & A & AC & C \\
\hline Riqueza de especies (S) & 9 & 17 & 8 \\
Uniformidad (equitivity) (E) & 0,53 & 0,61 & 0,34 \\
Índice de Simpson (DSp) & 0,38 & 0,28 & 0,70 \\
Índice de Shannon (H') & 1,17 & 1,72 & 0,71 \\
\hline
\end{tabular}

A, exclusivamente con larvas de Ae. albopictus; AC, larvas de Ae. albopictus y larvas de Chironominae sp. y Culex quinquefasciatus o Limatus durhamii; C, larvas de Chironominae sp. y Cx. quinquefasciatus o Li. durhamii; y NA, exhuvias de pupas o larvas de culícidos diferentes a Ae. albopictus.

encontraron correlaciones significativas con las poblaciones de otras larvas de Chironominae sp. $(\rho=-0,165, a=0,05), C x$. quinquefasciatus $(\rho$ $=0,093, a=0,05)$ y Li. durhamii $(\rho=-0,016, a=0,05)$ (figura 2a). Los criaderos AC se relacionaron más con depósitos de alta diversidad y riqueza de especies que los criaderos $C$, en los que habían moluscos y colémbolos en los márgenes del criadero como raspadores y recolectores (figura 2b).

Los criaderos $C$ presentaron los valores más altos de dominancia, por la presencia de poblaciones de Chironominae sp. (cuadro 3), pero no hubo una composición de macroinvertebrados que definiera esta clase de criaderos, como lo revela el valor de uniformidad (equitivity). El dendrograma de clasificación en la figura $2 \mathrm{~b}$ muestra las agrupaciones existentes entre los criaderos con larvas de Ae. albopictus y el componente de macroinvertebrados. Asimismo, los criaderos NA que no presentaron macroinvertebrados están más relacionados con los criaderos $\mathrm{C}$.

\section{Plancton}

Entre los organismos registrados en los criaderos con Ae. albopictus, se encontraron correlaciones significativas con bacilarofíceas $(\rho=0,888$, 


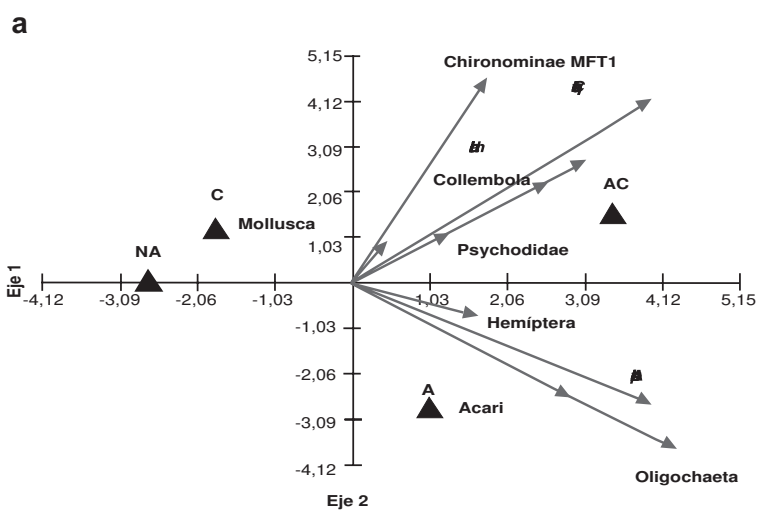

b

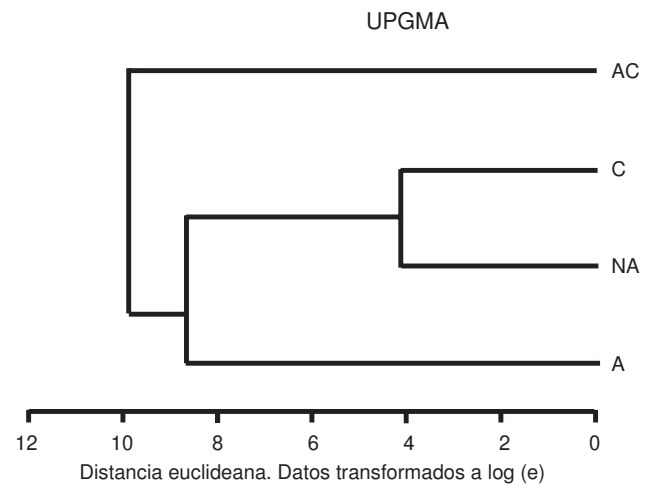

Figura 2. (a) Análisis de componentes principales a partir de la composición de macroinvertebrados presentes por clases de criaderos (el 99,1\% de la variación es explicada en los dos primeros componentes). (b) Dendrograma de clasificación a partir de la composición de macroinvertebrados presentes por clases de criaderos.

A, exclusivamente con larvas de Ae. albopictus; AC, larvas de Ae. albopictus y larvas de Chironominae sp. y Culex quinquefasciatus o Limatus durhamii; C, larvas de Chironominae sp. y Cx. quinquefasciatus o Li. durhamii; y NA, exhuvias de pupas o larvas de culícidos diferentes a Ae. albopictus.

Cuadro 4. Índices de diversidad por clases de criaderos a partir de las abundancias relativas del plancton.

\begin{tabular}{lcccc}
\hline Tipo de criadero & A & AC & C & NA \\
\hline Riqueza de especies (S) & 14 & 9 & 6 & 9 \\
Uniformidad (equitivity) (E) & 0,77 & 0,75 & 0,91 & 0,92 \\
Índice de Simpson (DSp) & 0,16 & 0,27 & 0,21 & 0,14 \\
\hline
\end{tabular}

A, exclusivamente con larvas de Ae. albopictus; AC, larvas de Ae. albopictus y larvas de Chironominae sp. y Culex quinquefasciatus o Limatus durhamii; C, larvas de Chironominae sp. y Cx. quinquefasciatus o Li. durhamii; y NA, exhuvias de pupas o larvas de culícidos diferentes a Ae. albopictus. $a=0,06)$, clorofíceas $(\rho=0,539, a=0,05)$ y cianofíceas $(\rho=0,487, a=0,05)$. La presencia de hojarasca en los criaderos AC aumenta la disponibilidad de alimento e incrementa las poblaciones de trozadores, filtradores, detritívoros y predadores, representados principalmente por poblaciones de rotíferos y copépodos. En los criaderos NA se presentó la proporción más alta de tecamebas (rizópodos), que son indicadores de aguas muy eutróficas.

En el cuadro 2 se muestran las abundancias relativas de plancton por clases de criaderos, donde las cianofíceas fueron las más abundantes en todos los criaderos. En el cuadro 4 se muestran los valores de riqueza, uniformidad (equitivity) y los índices de dominancia de Simpson y de diversidad de Shannon-Wiener; en los criaderos positivos para Ae. albopictus, hay mayor riqueza de especies y menor índice de uniformidad, característicos de los criaderos recién establecidos, en los que la cantidad de individuos y la presencia de especies productoras fue mayor. Debido a la competencia por espacio y recursos, aumenta la tasa de extinción en el hábitat, en los criaderos AC y C. Los valores de dominancia en todos los criaderos fueron bajos, debido principalmente a que la mayoría de sus componentes fueron productores.

Según el análisis de componentes principales, los criaderos A se caracterizaron por presentar abundancia en poblaciones de cianofíceas, otros organismos productores y diatomeas (bacillarofíceas), que no toleran niveles altos de nutrientes, lo cual puede ser sustentado por los bajos valores de conductividad registrados. Los criaderos $A C$ se relacionaron con poblaciones de consumidores primarios y secundarios, como son rotíferos, copépodos y cladóceros (figura 3a). En el dendrograma de clasificación se observa la agrupación de los criaderos $\mathrm{C}$ y NA, separado de los criaderos AC y, por último, de los criaderos A (figura $3 b$ ).

\section{Discusión}

Al igual que lo reportado por otros autores en diferentes partes del mundo, los criaderos de Ae. albopictus en Leticia se encontraron más frecuentemente en el peridomicilio (20), eran 
de tamaño mediano o pequeño, de plástico o metal $(21,22)$ y presentaban conductividad baja; estos valores dan un indicio de que los criaderos tienen una productividad y una diversidad altas, debido a las altas concentraciones de oxígeno disuelto y la baja toxicidad. Por otra parte, aunque la turbidez promedio fue baja, se registraron criaderos con valores más altos a los que se han reportado en otros estudios (23). La mayoría estaban a la sombra o parcialmente expuestos al sol (24), pero dos de ellos que eran de clase $A C$ tenían exposición directa al sol $(25,26)$. Esta preferencia se puede explicar porque, a pesar de que los huevos de $A e$. albopictus son resistentes a la desecación, es probable que en los criaderos pequeños y

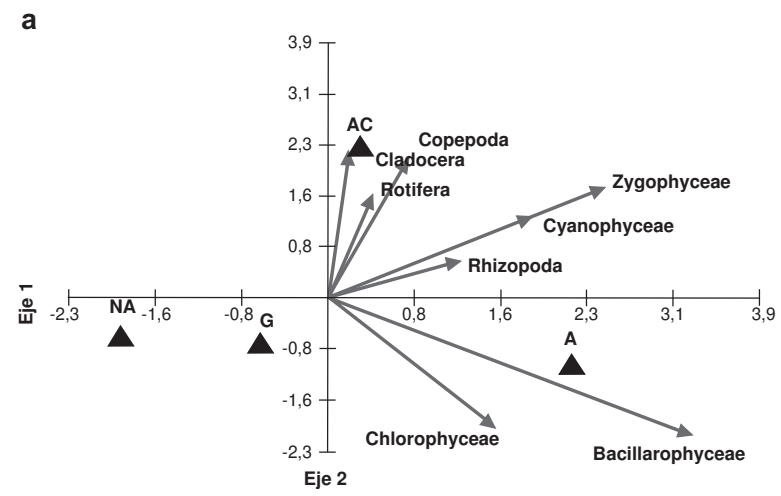

b

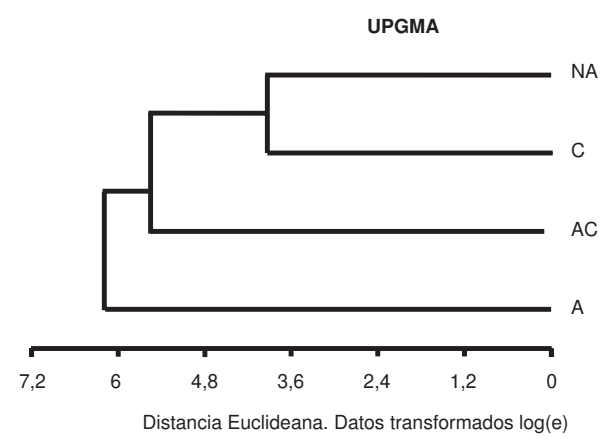

Figura 3. (a) Análisis de componentes principales a partir de la composición de plancton presente por clases de criaderos. (b) Dendrograma de clasificación a partir de la composición de plancton presente.

A, exclusivamente con larvas de Ae. albopictus; AC, larvas de Ae. albopictus y larvas de Chironominae sp. y Culex quinquefasciatus o Limatus durhamii; C, larvas de Chironominae sp. y Cx. quinquefasciatus o Li. durhamii; y NA, exhuvias de pupas o larvas de culícidos diferentes a Ae. albopictus. metálicos la temperatura se incremente a niveles que impidan la supervivencia y el desarrollo de las larvas; además, la densidad aumenta al disminuir el agua y, por ende, hay un incremento en la competencia por recurso y una mayor depredación (27-29).

La supervivencia de las larvas se puede afectar también por sustancias disueltas que se encuentren en al agua Iluvia, por metabolitos tóxicos de organismos en los depósitos o por algas presentes en el criadero (30-32), pero para la supervivencia de Ae. albopictus en un criadero el factor más importante parece ser la ausencia de competidores y depredadores. Por otro lado, la presencia de oligoquetos (naididos) en las criaderos $A$, es un indicador de la abundancia y diversidad de especies productoras y la baja presencia de consumidores, como depredadores, raspadores o recolectores, característicos de ambientes acuáticos más complejos y con mayor tiempo de establecimiento (31). En los criaderos NA hubo una escasa abundancia de consumidores, como rotíferos y copépodos, que puede estar relacionada con la poca materia en partículas, pero, se encontraron tecamebas (rizópodos), las cuales pueden presentarse por el aumento de bacterias.

Los parámetros bióticos y abióticos hallados muestran que las larvas de Ae. albopictus colonizan aguas recién estancadas, donde la competencia interespecífica no es alta y hay suficiente disponibilidad de recurso. A medida de que los criaderos se vuelven eutróficos, lo cual se refleja con la disminución del oxígeno disuelto, se establecen otras poblaciones de dípteros, lo que origina los criaderos $A C$, y la abundancia de larvas de Ae. albopictus disminuye. Esta preferencia de colonización por aguas recién estancadas es una ventaja potencial sobre las poblaciones de otros culícidos que se instauren posteriormente, porque pueden explotar los recursos de alimento y no ser afectadas por la contaminación del medio $(33,34)$.

Al excluir el volumen del criadero entre las variables por correlacionar, los criaderos AC y $A$ se relacionaron más estrechamente que los criaderos C y NA. La separación entre los 
criaderos C y NA puede deberse a que estos tienen un mayor tiempo de establecimiento $y$, por tanto, mayor eutrofización.

Entonces, la posible sucesión de las poblaciones presentes en los criaderos la podemos definir así: las poblaciones de Ae. albopictus colonizan los depósitos recién establecidos, este hecho se correlaciona con los valores altos de índice de infestación de depósitos en las épocas de mayor pluviosidad (12); posteriormente, se establecen quironómidos y otros culícidos, que generan competencia por recursos, la presencia de predadores o sustancias y plancton tóxicos en el criadero, las cuales posiblemente reducen las poblaciones de Ae. albopictus hasta que desaparecen.

Sin embargo, al analizar los criaderos por los morfotipos presentes para determinar el nivel de competencia, se encontró que, en presencia de quironómidos, las poblaciones de Ae. albopictus no se redujeron considerablemente, lo que significa que el recurso por el que compiten posiblemente no es el mismo, mientras que, en presencia de larvas de otros culícidos, las de $A e$. albopictus no fueron abundantes, posiblemente por competencia o por depredación, ya que las larvas de Li. durhami, especies de las familias Veliidae y Gerridae y algunos copépodos se han reportado como depredadores y, las larvas de ácaros, como parásitos de Ae. albopictus (35-38).

Uno de los modelos que se puede aplicar a este tipo de hábitats acuáticos con poblaciones locales conectadas entre sí por eventos de migración, es la "teoría de la dinámica de las metapoblaciones". Una metapoblación, por lo tanto, puede definirse como una "población de poblaciones": un grupo de poblaciones locales conectadas por migraciones, en las que los principales procesos de población involucrados son las migraciones, las colonizaciones y las extinciones (39).

En Leticia, las poblaciones locales se conectarían a través de la dispersión de un pequeño número de hembras migrantes que colonizan nuevos hábitats en depósitos recién establecidos o se juntan con poblaciones preexistentes. Además, en este modelo la estructura espacial es importante por la discontinuidad que existe entre ellos, ya que diferentes grupos de individuos de la misma especie pueden crecer en unidades de hábitat aisladas y, por lo tanto, las dinámicas de población son casi independientes. Entonces, la persistencia de una metapoblación, a largo plazo, depende de la existencia de varias poblaciones relativamente próximas, que garanticen el flujo de individuos y la disponibilidad de hábitats. Sin embargo, para corroborar esta hipótesis se necesitan nuevos estudios.

\section{Agradecimientos}

A la Secretaría de Salud Departamental del Amazonas, a la Universidad Nacional de Colombia y a la población de Leticia, por su apoyo para la realización de este estudio.

\section{Conflicto de intereses}

Los autores declaramos que no existe conflicto de intereses sobre los resultados presentados en este artículo.

\section{Financiación}

Este trabajo lo financiaron la Universidad Nacional de Colombia y la Secretaría de Salud Departamental del Amazonas.

\section{Referencias}

1. Forattini OP. Identificão de Aedes (Stegomyia) albopictus no Brasil. Rev Saúde Pública. 1986;20:244-5.

2. Vélez ID, Quiñones M, Suárez M, Olano V, Murcia LM, Correa E, et al. Presencia de Aedes albopictus en Leticia, Amazonas, Colombia. Biomédica. 1998;18:192-8.

3. Suárez M. Aedes albopictus (Skuse) (Diptera, Culicidae) en Buenaventura, Colombia. Inf Quinc Epidemiol Nac. 2001;6:221-4.

4. Cuéllar-Jiménez ME,Velásquez-EscobarOL, GonzálezObando R, Morales-Reichman CA. Detección de Aedes albopictus (Skuse) (Diptera: Culicidae) en la ciudad de Cali, Valle del Cauca, Colombia. Biomédica. 2007;27:273-9.

5. Gratz NG. Critical review of the vector status of Aedes albopictus. Med Vet Entomol. 2004;18:215-27.

6. Serufo JC, Montes de Oca HM, Tavares VA, Souza AM, Rosa RV, Jamal MC, et al. Isolation of dengue virus type 1 from larvae of Aedes albopictus in Campos Altos City, State of Minas Gerais, Brazil. Mem Inst Oswaldo Cruz. 1993;83:503-4. 
7. Ibañez-Bernal S, Briceño B, Mutebi SP, Argot E, Rodríguez G, Martínez-Campos C, et al. First record in America of Aedes albopictus naturally infected with dengue virus during the 1995 outbreak at Reynosa, México. Med Vet Entomol. 1997;11:305-9.

8. Holick J, Kile A, Ferraro W, Delaney R, Iwaseczko M. Discovery of Aedes albopictus infected with West Nile Virus in Southeastern Pennsylvania. J Am Mosq Control Assoc. 2002;18:131.

9. Méndez F, Barreto M, Arias JF, Rengifo G, Muñoz J, Burbano ME, et al. Human and mosquito infections by dengue viruses during and after epidemics in a dengueendemic region of Colombia. Am J Trop Med Hyg. 2006;74:678-83.

10. Olano V. Vigilancia entomológica: un componente en salud pública. Inf Quinc Epidemiol Nac. 1999;4:273-4.

11. Instituto Geográfico Agustín Codazzi. Atlas Básico de Colombia. Bogotá, D.C.; División de Difusión Geográfica; 1989.

12. Secretaría de Salud Departamental. 2001-2003. Informes de levantamiento de índice aédico en el municipio de Leticia. Leticia: Gobernación del Amazonas; 2004.

13. Berríos V, Sielfeld W. Superclase crustácea, crustáceos continentales. Programa de Biodiversidad, Universidad Arturo Prat lquique, Chile. Fecha de consulta: 12 de julio de 2005. Disponible en: http://www.insectos.cl/pdf/ CRUSTACEA.pdf.

14. McAlpine J, Peterson B, Shewell G, Teskey H, Vockeroth J. Manual of Nearctic Diptera. Quebec: Canadian Government Publishing Centre; 1993.

15. Merritt RW, Cummins KW. An introduction to the aquatic insects of North America. Third edition. Dubuque, lowa: Kendall/Hunt Publishing Company; 1996.

16. Milligan $\mathbf{M}$. Identification manual for the aquatic Oligochaeta of Florida. Sarasota, Florida: Freshwater Oligochaetes; 1997.

17. Peckarsky BL, Fraissinet PR, Penton MA, Conklin DJ. Freshwater macroinvertebrates of northeastern North America. Ithaca, New York: Cornell University Press; 1990

18. Thorp J, Covich A. Ecology and classification of North American Freshwater Invertebrates. 2nd edition. San Francisco: Academic Press; 2001.

19. La Casse WJ, Yamaguti S. Mosquito fauna of Japan and Korea. Washington, D.C.: USAF School of Aviation Medicine Air University; 1950.

20. Chareonviriyaphap T, Akratanakul P, Nettanomsak S, Huntamai S. Larval habitats and distribution patterns of Aedes aegypti (Linnaeus) and Aedes albopictus (Skuse), in Thailand. Southeast Asian J Trop Med Public Health. 2003;34:529-35.
21. Simard F, Nchoutpouen E, Toto JC, Fontenille D. Geographic distribution and breeding site preference of Aedes albopictus and Aedes aegypti (Diptera: Culicidae) in Cameroon, Central Africa. J Med Entomol. 2005;42:726-31.

22. Foratini OP, Kakitani I, Mureb MA, De Rezende L. Produtividade de criadouro de Aedes albopictus em ambiente urbano. Rev Saúde Pública. 1997;31:545-55.

23. Sehgal SS, Pillot MK. Preliminary studies on the chemical nature of mosquito-breeding waters in Dehli. Bull World Health Organ. 1970;42:647-50.

24. Carrieri M, Bacchi M, Bellini R, Maini S. On the competition occurring between Aedes albopictus and Culex pipiens (Diptera: Culicidae) in Italy. Environ Entomol. 2003;32:1313-21.

25. Devi NP, Janhari RH. Mosquito species associated within some western Himalayas phytogeographic zones in the Garhwal region of India. J Insect Sci. 2007;7:1-10.

26. Alto B, Juliano S. Precipitation and temperature effects on populations of Aedes albopictus (Diptera: Culicidae): implications for range expansion. J Med Entomol. 2001;38:646-56.

27. Sota T, Mogi M. Survival time and resistance to desiccation of diapauses and non-diapause eggs of temperate Aedes (Stegomyia) mosquitoes. Entomol Exp Appl. 1992;63:151-61.

28. Sunahara T, Mogi M. Variability of intra and interespecific competitions of bamboo stump mosquito larvae over small and large spatial scales. Oikos. 2002;97:87-96.

29. Calado D, Navarro MA. Avaliaçâo da influência da temperatura sobre o desenvolvimiento de Aedes albopictus. Rev Saúde Pública. 2002;36:173-9.

30. Estrada-Franco J, Craig G Jr. Biología, relaciones con enfermedades y control de Aedes albopictus (Cuaderno técnico No. 42). Washington D. C.: Organización Panamericana de la Salud; 1995.

31. Learner MA, Lockhead G, Hughes BD. A review of the biology of British Naididae (Oligochaeta) with emphasis on the lotic environment. Freshwater Biol. 1978;8:357-75.

32. Marten G. The potential of mosquito-indigestible phytoplankton for mosquito control. J Am Mosquito Control Assoc. 1987;3:105-6.

33. Sunahara T, Ishizaka K, Mogi M. Habitat size: determining the opportunity for encounters between mosquito larvae and aquatic predators. J Vector Ecol. 2002;27:8-20.

34. Roldán G. Fundamentos de limnología neotropical. Colección Ciencia y Tecnología Universidad de Antioquia. Medellín: Editorial Universidad de Antioquia; 1992.

35. Sunahara T, Mogi M. Priority effects of bamboo-stump mosquito larvae: Influences of water exchange and leaf litter input. Ecol Entomol. 2002;27:346-54. 
36. Lopes J. Ecologia de mosquitos (Diptera, Culicidae) em criadouros naturais e artificiais de area rural do norte do Paraná, Brasil. VIII. Influência das larvas predadoras (Toxorhynchites sp., Limatus durhamii e Culex bigoti) sobre a população de larvas de Culex quinquefasciatus e Culex eduardoi. Rev Bras Zool. 1999;16:821-6.

37. Smith B, Mclver S. The patterns of mosquito emergence (Diptera: Culicidae; Aedes spp.); their influence on host selection by parasitic mites (Acari: Arrenuridae: Arrenurus spp.). Can J Zool. 1984;62:1106-13.
38. Nam VS, Yen NT, Holynska M, Reid JW, Kay BH. National progress in dengue vector control in Vietnam: Survey for Mesocyclops (Copepoda), Micronecta (Corixidae), and fish as biological control agents. Am J Trop Med Hyg. 2000;62:5-10.

39. Marini O, Parentoni R. La teoría de las metapoblaciones. Nuevos principios de la biología de la conservación. Ciencia Hoy. 2001;11:7-8. 